\title{
ACROMION NON-UNION WITH ASSOCIATED POSTERIOR GLENOID LABRAL TEAR
}

Karthik Neelakandan'1, Jai Thilak ${ }^{2}$

\section{HOW TO CITE THIS ARTICLE:}

Karthik Neelakandan, Jai Thilak. "Acromion Non-Union with Associated Posterior Glenoid Labral Tear". Journal of Evolution of Medical and Dental Sciences 2014; Vol. 3, Issue 37, August 21; Page: 9585-9588,

DOI: $10.14260 /$ jemds/2014/3240

ABSTRACT: Acromion fractures are often associated with high velocity trauma. These fractures are relatively rare injuries with the reported incidence being $0.1 \%$ of skeletal fractures in the literature. The pathology of Acromion non-union with associated soft tissue lesions and their treatment have been sparsely reported in the literature. Our case is that of a forty year old male with complaint of pain and restriction of his left shoulder for 7 months following a road traffic accident. He was diagnosed to have an Acromion non - union with an associated posterior glenoid labral tear. We report the successful treatment of the acromion non-union and the associated posterior glenoid labral tear by a combination of Arthroscopic and open reduction procedures.

KEYWORDS: Acromion, Non-union, Glenoid labrum, acromion surgery.

INTRODUCTION: Acromion fractures are relatively uncommon injuries accounting to around 8-10\% of scapular fractures. ${ }^{1}$ Scapula fractures by themselves are rare representing $1 \%$ of all skeletal fractures and $3-5 \%$ of shoulder fractures.2, 3 These fractures are high energy fractures with the incidence of associated injuries ranging from $80-95 \% .4$

CASE REPORT: A Forty year old male presented to our department with complaints of pain in the left shoulder with strenuous activities \& minimal restriction of shoulder movement, for duration of 7 months. He gave a history of Road traffic accident and had sustained injury to the left shoulder 7 months ago following which he was treated in a peripheral hospital by means of sling immobilization.

His pain reduced in intensity but he still had difficulty in strenuous activities and had restricted himself from overhead activities. Examination of the shoulder revealed a bony crepitus over the acromion on abduction with the restriction of abduction to 110 degrees, internal rotation restricted to the level of L5 and with full range of external rotation.

Roentgenographic viewing of the shoulder with Anteroposterior, Axillary and supraspinatus outlet Views along with CT scan demonstrated a displaced Non - Union of the left Acromion with the fragment reducing the subacromial space. Magnetic resonance imaging of the shoulder showed an associated Posterior glenoid labral avulsion \& Supraspinatus tendinitis. 


\section{CASE REPORT}

Anteroposterior \& Supraspinatus outlet views revealing the displaced Acromion Non - Union.
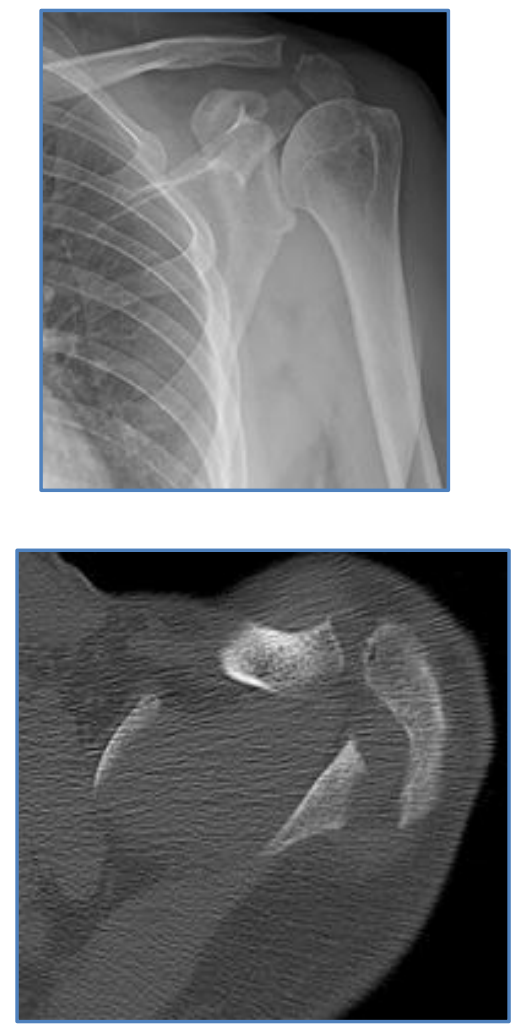

CT picture of the displaced acromion fragment
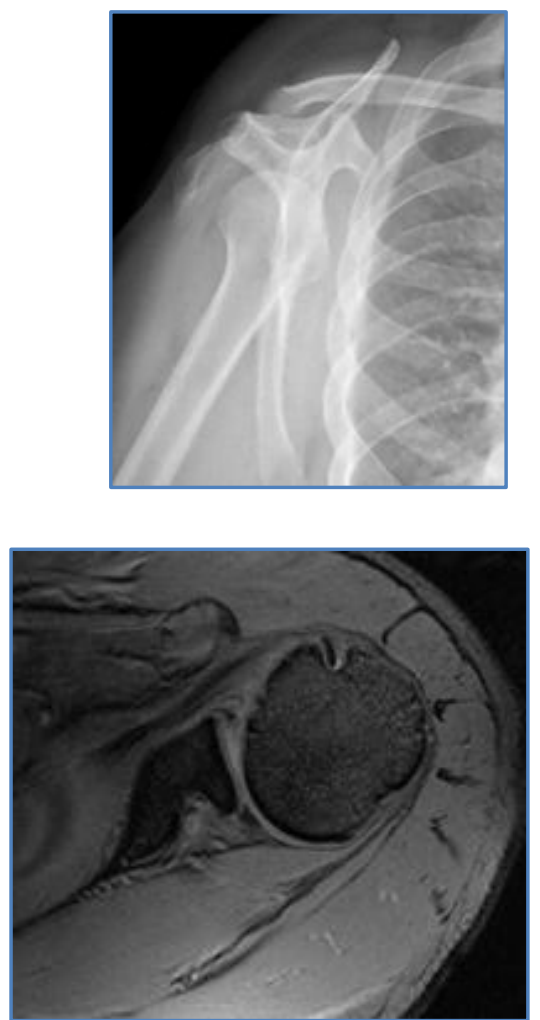

MRI - labral avulsion

Routine blood investigations were found to be normal; the patient was planned for surgical intervention. Arthroscopic evaluation of the shoulder was made and labral avulsion was stabilized with $2.8 \mathrm{~mm}$ suture anchors initially and acromion was approached through posterior approach along langhans lines and acromion non - union was fixed with $3.5 \mathrm{~mm}$ Reconstruction plate and screws along with iliac crest bone grafting. Post operatively patient was immobilized in an Arm sling for a period of 3 weeks following which pendulum exercises \& passive mobilization were initiated, range of movement were initiated actively from 6 weeks.

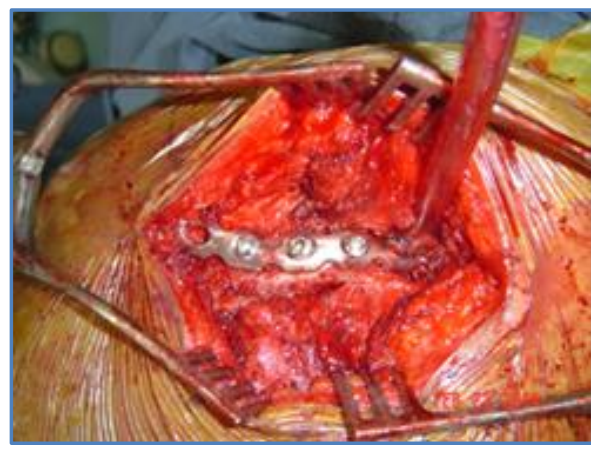

Intraoperative picture

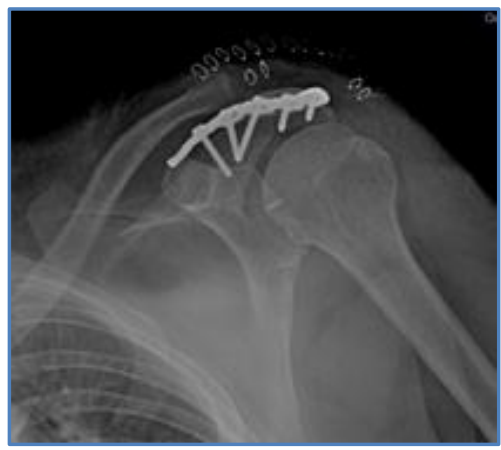

Immediate post-operative $\mathrm{x}$-ray view 
Patient was followed with clinical and radiological evaluation at 3 months, 6 months, 9months \& 1 year. The patient was evaluated at $1 \mathrm{yr}$. and 6months post-op and has regained full range of movements of the shoulder with no complaints of pain on extreme abduction \& overhead activities. We had obtained an informed consent from the patient for the purpose of this report.
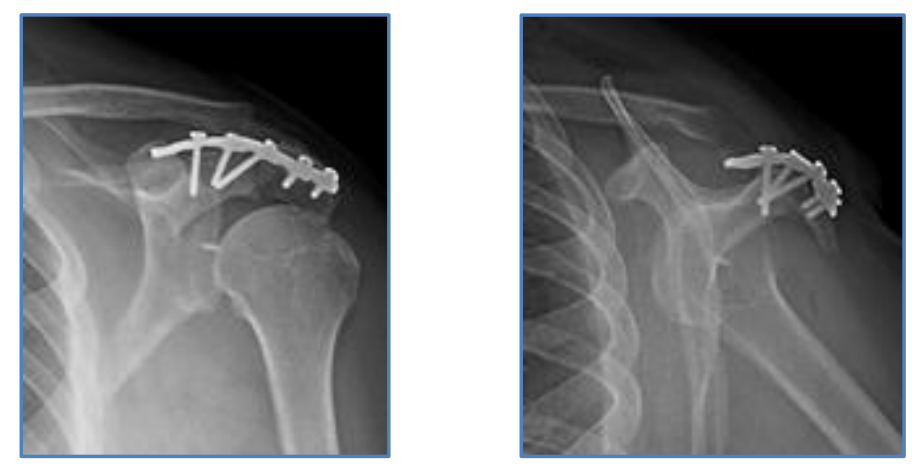

Follow - up x-ray views at 1 year and 6 months

DISCUSSION: Acromion fractures are relatively rare as being a stronger bone by itself; it involves considerable energy for an acromion fracture to occur primarily without any underlying pathology of the acromion. The mechanism of injury may involve either a direct injury over acromion, superior dislocation of humeral head ${ }^{6}$ or inferior dislocation with luxatio erecta. ${ }^{7}$

Since these injuries are involved in high velocity trauma, there are a high percentage of associated injuries in these patients which take priority in the management protocol. As acromion fractures are usually minimally displaced most of these have been treated by non - surgical methods.

But these fractures have to be closely followed as they have a tendency to get displaced due to the pull of the deltoid over it. The complications occurring in a conservatively managed acromion fracture have been reported from pain, limitation of movement, rotator cuff tears and humeral head migration.

Studies pertaining to acromion fractures are scarcely found in the literature with only a few cases of acromion non-union have been reported and the treatment guidelines for these fractures and associated injuries have been limited. Since considerable force is required to cause an acromion to fracture there may be an associated injury to the soft tissues surrounding it as the force gets dissipated into these structures.

The classification for the treatment of acromion fracture by Kuhn et.al ${ }^{8}$ where surgical indication is proposed for type III injuries of fracture of acromion with space reduction and the proposition by Goss (1993) ${ }^{9}$ of double disruption of the superior shoulder suspensory complex can result in an unstable anatomical situation involving the acromion and should be considered for operative treatment.

This case study shows the rare necessity of surgical treatment for an acromion fracture in active patients with successful treatment of the associated labral injury. 


\section{REFERENCES:}

1. Wilber MC, Evans EB. Fractures of the scapula: an analysis of forty cases and a review of the literature. J Bone Joint Surg [Am] 1977; 59-A: 358-62.

2. Fernandes MR et al. Fracture of the acromion with arterial lesion. J Vasc Bras. 2010; 9 (2): 95-100.

3. Imatani RJ. Fractures of the scapula: A review of 53 fractures. J Trauma 15: 473-478, 1975.

4. McGahan JP, Rab GT, Dublin A. Fractures of the scapula. J Trauma 20:880-883, 1980.

5. Goss TP. Fractures of the glenoid cavity. J Bone Joint Surg (Am) 1992: 74-A: 299-305.

6. Rockwood C A, Green D P. Fractures. Ed. 2, pp. 718-719. Philadelphia, J. B. Lippincott, 1984.

7. Pavlov Helene, Freiberger R H. Fractures and Dislocations about the Shoulder. Sem Roentgenol., 13: 85-96, 1978.

8. Kuhn JE, Blasier RB, Carpenter JE. Fractures of the acromion process: a proposed classification system. J Orthop Trauma. 1994; 8: 6-13.

9. Goss T P. Double disruptions of the superior shoulder suspensory complex. J Orthop Trauma 1993; 7: 99-106.

\section{AUTHORS:}

1. Karthik Neelakandan

2. Jai Thilak

\section{PARTICULARS OF CONTRIBUTORS:}

1. Assistant Professor, Department of Orthopaedics/Arthroscopy, SRM MCH \& RC.

2. Clinical Professor, Department of Orthopaedics/Arthroscopy, AIMS, Kochi.

\section{NAME ADDRESS EMAIL ID OF THE} CORRESPONDING AUTHOR:

Dr. Karthik Neelakandan, Assistant Professor, SRM Medical College.

Email: dr_nk2001@yahoo.co.in

Date of Submission: 11/08/2014.

Date of Peer Review: 12/08/2014.

Date of Acceptance: 18/08/2014.

Date of Publishing: 19/08/2014. 